\title{
Studies on Ultrasonic Initiated Copolymerization of Styrene and Acrylate Series
}

\author{
Jiang LiU, Keqiang CHEN, and Zhili Li \\ State Key Lab. of Polymer Material \& Engineering, Research Institute of Sichuan University, \\ Chengdu 610065, People's Republic of. China
}

(Received June 14, 1999)

\begin{abstract}
Experiments with styrene (St) and acrylate monomer [methyl acrylate (MA), ethyl acrylate (EA) and butyl acrylate (BA)] prove that copolymerization can be initiated in two monomers with intense ultrasound. Within the experimental range, the yields of copolymer increase with rise of ultrasonic intensity, reaction time, temperature and the amount of ion surfactants (sodium dodecyl sulfate (SDS), hexadecyltrimethyl ammonium bromide (CTAB)), but decrease with increasing concentration of comonomers. The comonomer ratio and surfactant types have considerable effect on the yield of copolymer. The yield of copolymer is $38.8 \%$ by irradiating $10 \% \mathrm{BA} / \mathrm{St}(1 / 1, \mathrm{v} / \mathrm{v})$ at $40^{\circ} \mathrm{C}$ with $600 \mathrm{w}$ for $1 \mathrm{~h}$. KEY WORDS Ultrasound / Copolymerization / Monomer /
\end{abstract}

Ultrasound has been applied for polymer synthesis since 1980 s. $^{1}$ Compared with general chemical method, ultrasonic polymerization has characteristics as follows : (1) accelerating chemical reactions or easing reaction conditions ; (2) lowering the requirement for reagent; (3) initiating polymerization without the addition of an initiator; (4) simplifying the procedures of synthesis; (5) synthesizing polymers unable to be obtained through general chemical methods. Therefore, ultrasound provides energy to modify chemical reactivity, energy, which is different from the commonly used heat, light, radiation etc. So far the vast majority of investigations have been carried out in systems containing homopolymers. Since 1985, several papers concerning the ultrasonically initiated polymerization of vinyl monomers appeared..$^{2-4}$ However, study on copolymerization in monomer-monomer system with ultrasound is little. This paper examines the ultrasonically initiated emulsion copolymerization of styrene and acrylate monomers (methyl acrylate, ethyl acrylate and butyl acrylate). The effects of ultrasonic intensity, reaction time, bulk temperature, ratio of comonomer, surfactant type, concentration of surfactant and comonomer on the yield of copolymer are systemically studied.

\section{EXPERIMENTAL}

\section{Material}

Methyl acrylate (MA), ethyl acrylate (EA), butyl acrylate (BA) and styrene (St) were vacuum-distilled to remove the hydroquinone inhibitor and refrigerated until use. The sodium dodecyl sulfate (SDS) surfactant was purified through twice crystallization in ethanol. Hexadecyltrimethyl ammonium bromide (CTAB) and octylphenol polyethylene oxide-10 (OP-10) were of analytical purity and used without further purification. The tetrahydrofuran (THF), acetone, ethanol, and ethyl acetate were of chemical purity grade.

\section{Apparatus}

As shown in Figure 1, a Sonics and materials ultrasonic generator $(20 \mathrm{KHz}$ Model VC-1500) was used with standard titanium horn to introduce ultrasound directly into the liquid. A compressed air cooled transducer was connected to the horn. The copolymerization was performed in a $120-\mathrm{ml}$ glass beaker surrounded by a circulating water bath. The top of the beaker was covered with rubber lid. Nitrogen gas was bubbled through the solution by a metal frit.

\section{Procedure}

The emulsion was prepared with $90.0-\mathrm{ml}$ distilled water, surfactant and the monomers with different monomer ratios $\left(V_{\mathrm{MA}} / V_{\mathrm{St}}, V_{\mathrm{EA}} / V_{\mathrm{St}}, V_{\mathrm{BA}} / V_{\mathrm{St}}\right)$ measured volumetrically. One variation was changed at a time when other variations remained constant to gain data for optimizing the reaction. Variables include changing the amount of surfactant $(0.36 \mathrm{~g}, 0.72 \mathrm{~g}, 1.08 \mathrm{~g}, 1.8 \mathrm{~g}$, $2.16 \mathrm{~g})$, monomer concentrations $(10 \%, 15 \%, 20 \%, 25 \%$, $35 \%)$, monomer ratio, bulk temperature $\left(15-40^{\circ} \mathrm{C}\right)$, acoustic intensity (as a percent of maximum output: $10 \%, 20 \%, 30 \%, 40 \%$ ).

The emulsion was introduced into the flask and deoxygenated by bubbling with dry, oxygen free nitrogen for 5 min. The ultrasound was switched on and a nitrogen atmosphere was maintained during sonication. After sonication, the beaker was removed and the emulsion was transferred to a $500-\mathrm{ml}$ flask. The product was coagulated by $200-\mathrm{ml}$ ethanol. The polymer was dissolved in THF and precipitated by a large amount of distilled

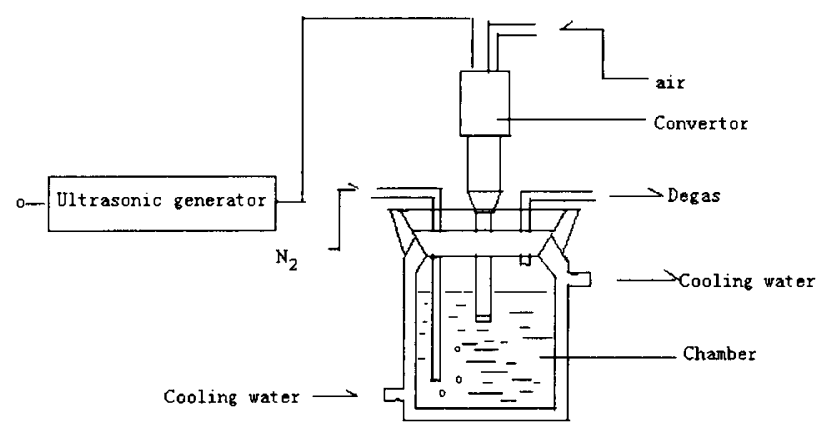

Figure 1. Ultrasonic reactor. 
water to remove surfactants. The reaction product was extracted with acetone and ethyl acetate for $48 \mathrm{~h}$ to remove unreacted monomers and homopolymers. The final copolymer was weighed and collected for further testing.

\section{Identification of Copolymer}

The purified polymer was analyzed by IR (Nicolet-FTInfrared Spectrometer). The glass transition temperature $\left(T_{\mathrm{g}}\right)$ of copolymer was determined with a Differential Scanning Calorimeter (DSC 2 c, Perkin Elmer), at a heating rate of $20^{\circ} \mathrm{C} \mathrm{min}{ }^{-1}$.

\section{RESULTS AND DISCUSSION}

Figure 2 is the IR spectrum of final purified copolymer. Wavenumbers and corresponding structures are shown in Table I, which indicates that the IR spectrum of the copolymer includes characteristic absorption of both polystyrene and polyethyl acrylate. DSC curve of the copolymer (Figure 3 ) suggests the structure of copolymer, which shows only one $T_{\mathrm{g}}\left(49.54^{\circ} \mathrm{C}\right)$, lower than $T_{\mathrm{g}}$ of PS $\left(100^{\circ} \mathrm{C}\right)$ and higher than $T_{\mathrm{g}}$ of $\operatorname{PEA}\left(-24^{\circ} \mathrm{C}\right)$.

Figure 4 represents the effect of change in ultrasonic intensity, which shows an increase in the percent yield as a function of ultrasonic intensity. The reason is that at a certain frequency, increase of ultrasonic intensity determines the magnitude of the cavitation area. Cavitation bubbles cannot be created until the ultrasonic intensity reaches a certain point known as cavitation threshold. After the cavitation threshold, with increase of sonic intensity (I), pressure and amplitude of ultrasound $\left(P_{\mathrm{a}}\right)$ in the medium increase, resulting in higher pressure in liquids $\left(P_{\mathrm{m}}, P_{\mathrm{m}}=P_{\mathrm{h}}+P_{\mathrm{a}}, P_{\mathrm{h}}\right.$ represents static pressure of fluid). The relationships between $P_{\mathrm{m}}$ and maximum temperature $\left(T_{\max }\right)$ and maximum pressure $\left(P_{\max }\right)$ and collapsing time of cavitation bubbles $(\tau)$ are shown as,

$$
\begin{gathered}
\tau=0.915 R_{\mathrm{m}}\left(\rho / P_{\mathrm{m}}\right)^{1 / 2}\left(1+P_{\mathrm{v}} / P_{\mathrm{m}}\right) \\
T_{\max }=T_{\text {bulk }}\left[P_{\mathrm{m}}(\gamma-1) / P_{\mathrm{v}}\right] \\
P_{\max }=P_{\mathrm{g}}\left[P_{\mathrm{m}}(\gamma-1) / P_{\mathrm{v}}\right]^{\gamma /(\gamma-1)}
\end{gathered}
$$

Where $P_{\mathrm{m}}$ is the pressure generated on collapse, $\gamma$ ra tio of specific heat capacities of the solvent vapor, and $P_{\mathrm{v}}$ vapor pressure of the solvent at temperature, $\rho$ liquid density, $R_{\mathrm{m}}$ maximum cavitation bubble radius, $P_{\mathrm{g}}$ initial pressure in cavitation bubbles and $T_{\text {bulk }}$ ambient temperature. Cavitational collapse creates drastic conditions inside the medium : temperatures of $2000-5000 \mathrm{~K}$ and pressures up to $1800 \mathrm{~atm}$ within the collapsing cavity. ${ }^{1}$ For example, in the water $\left(25^{\circ} \mathrm{C}\right)$ system keeping with nitrogen atmosphere, the estimated values of $P_{\max }$, $T_{\max }$, and $\tau$ are respectively $9.80 \times 10^{7} \mathrm{~Pa}, 4290 \mathrm{~K}$ and $1 \mu \mathrm{s} .5$

Equations $1-3^{5}$ mean that the increase of $P_{\mathrm{m}}$ is helpful to obtain shorter $\tau$, higher $T_{\max }$ and $P_{\max }$, respectively, which intensify the extent of cavitation collapse and facilitate the dispersion of monomers and free radicals. Therefore the yield of copolymer increases with sonic intensity.

Figure 5 shows the time dependence of ultrasonically

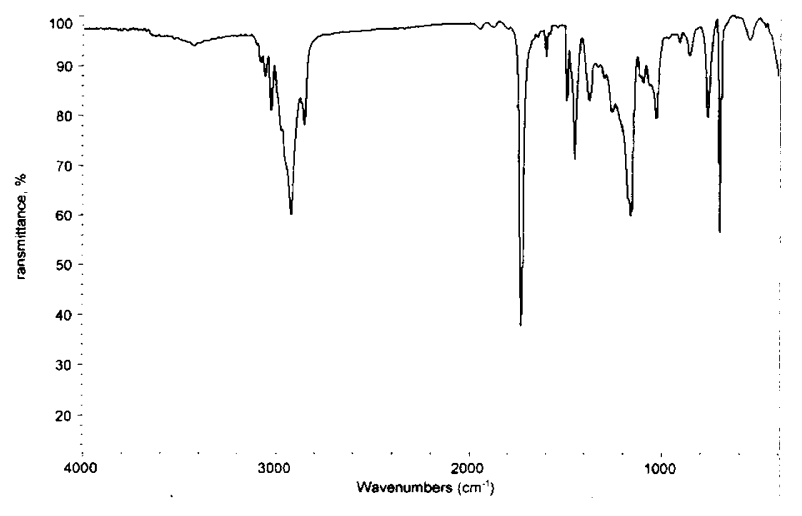

Figure 2. IR spectrum of PS-co-PEA copolymer.

Table I. IR assignment of PS-co-PEA copolymer

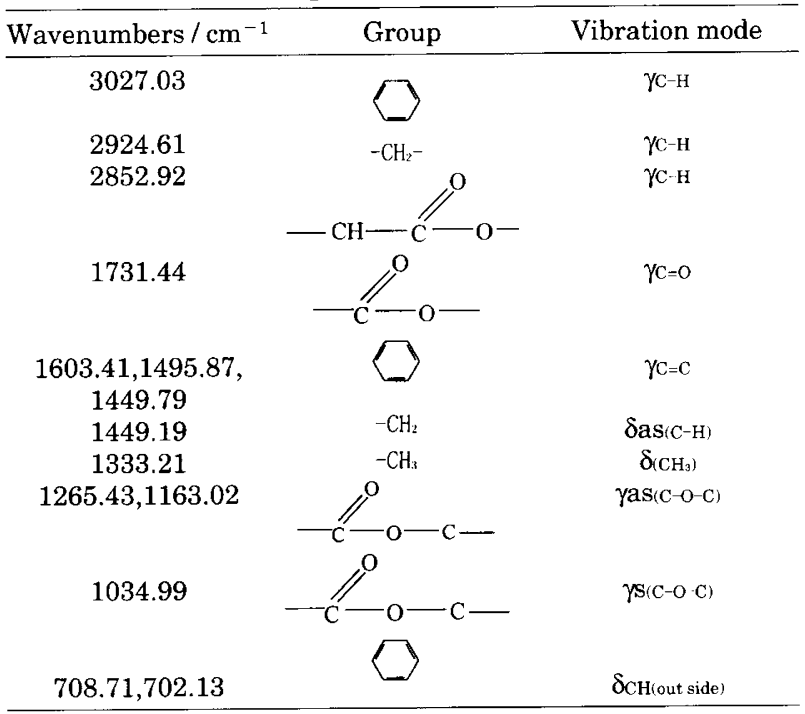

initiated polymerization. The longer the reaction time, the higher the yield of the copolymer is. However, increase in yield slows with time, suggesting that copolymerization of monomers and degradation of polymers competes with each other during the sonication. At the beginning, copolymerization dominates because the concentration of monomer is much higher than that of polymer. The degradation starts with increase of polymer. During the degradation, although the number of free radicals in the system remains, the dispersing rate of free radicals falls with increase of viscosity and decrease of concentration of monomers.

The relationship between bulk temperature and yield of copolymer is illustrated in Figure 6. Two temperatures exist in the system of ultrasonic copolymerization : $T_{\max }$ (the maximum temperature) and $T_{\text {bulk }}$ (bulk temperature). The most widely accepted treatment assumes very high temperatures and pressures during an adiabatic bubble collapse. On simplified treatment, $T_{\max }$ is given by eq 2 . Clearly, this is sufficiently high to dissociate solvent vapor entering the bubble, leading to bond breakage and radical formation. When $T_{\text {bulk }}$ is relatively low, the content of solvent vapor is low, resulting in a low value of $P_{\mathrm{v}}$ and a higher $T_{\max }$. At this time, free radicals with high energy tend to combine with each other to form oligomer, which results in decrease of number of 


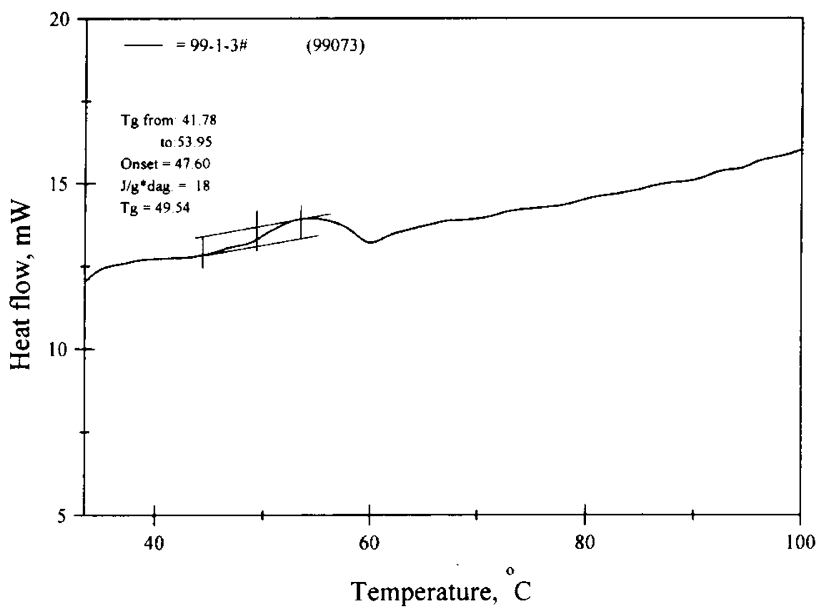

Figure 3. DSC spectrum of PS-co-PEA copolymer.

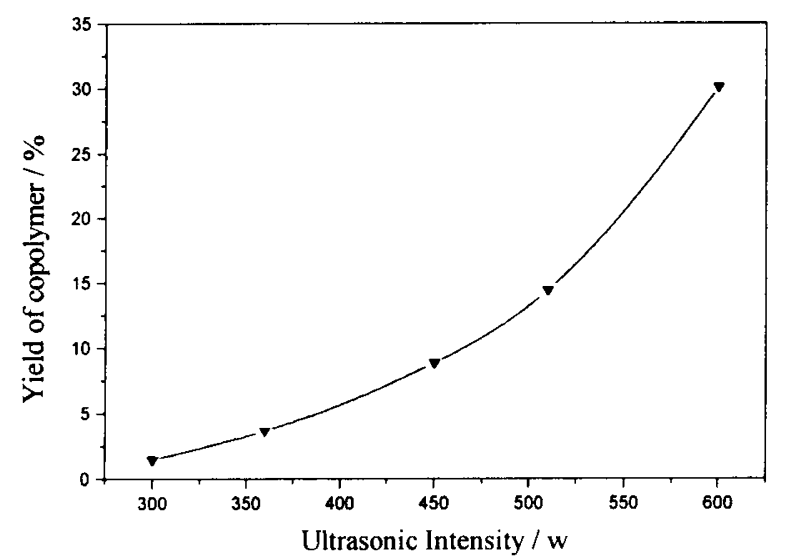

Figure 4. Effects of ultrasonic intensity on yield of PS-co-PEA at $18^{\circ} \mathrm{C}$ (conc. of comonomer : $10 \%$, conc. of SDS : $2.5 \%$, irradiation time : $\left.1 \mathrm{~h} ; V_{\mathrm{EA}} / V_{\mathrm{St}}=1\right)$.

radicals able to disperse out of the cavitation bubble to initiate polymerization. When $T_{\text {bulk }}$ increases, $P_{\mathrm{v}}$ increases quickly which facilitates the formation of cavitation bubbles. Higher $P_{\mathrm{v}}$ leads to the fall of $T_{\max }$, but enough to generate radicals. The generated radicals with a medium energy prefer to disperse out of the cavitation bubbles and initiate copolymerization. Therefore the yield of copolymer increases with bulk temperature.

Kruus Riesz reports ${ }^{2}$ that St, EA, and $\mathrm{H}_{2} \mathrm{O}$ can form free radicals under the cavitating of ultrasound. However, the yields of the copolymerization in systems respectively containing only pure monomers (St/EA), monomer/surfactant, monomer/water are almost naught. This suggests that whether free radicals can initiate polymerization is dependent on their longevity and reactivities. Only those free radicals which exist long enough to disperse out of the cavitation bubbles can initiate the copolymerization.

Experiments prove that the addition of anion surfactant SDS and cation surfactant CTAB facilitates the dispersion of free radicals from cavitation bubbles to the monomer solution. But non-ion surfactant OP-10 has no such effect, as shown in Figure 7 and Table II. The reason is that ion surfactant is able to form micelles containing double-electron layers in monomer solution. In

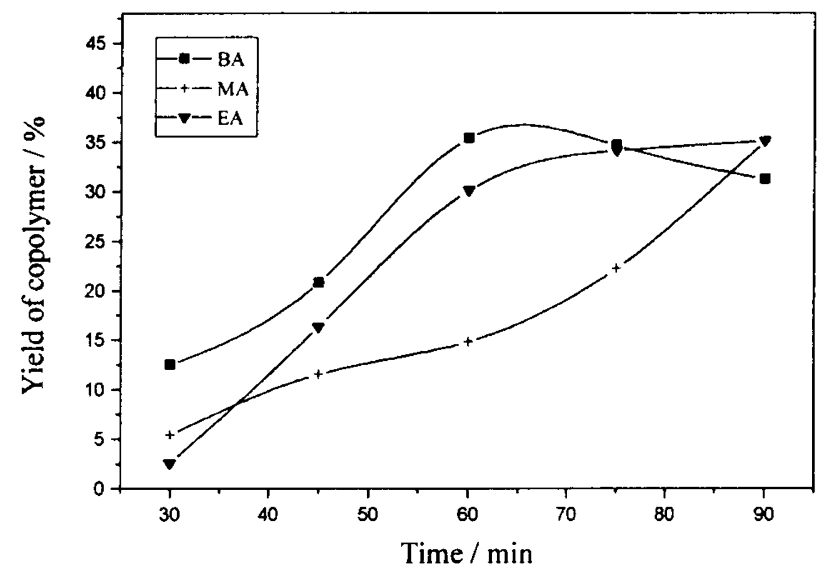

Figure 5. Effects of irradiation time on yield of copolymer at 25 ${ }^{\circ} \mathrm{C}$ (sonic intensity : $600 \mathrm{w}$; conc. of comonomer: 10\%; conc. of $\left.\operatorname{SDS}: 2.5 \% ; V_{\mathrm{A}} / V_{\mathrm{St}}=1\right)$.

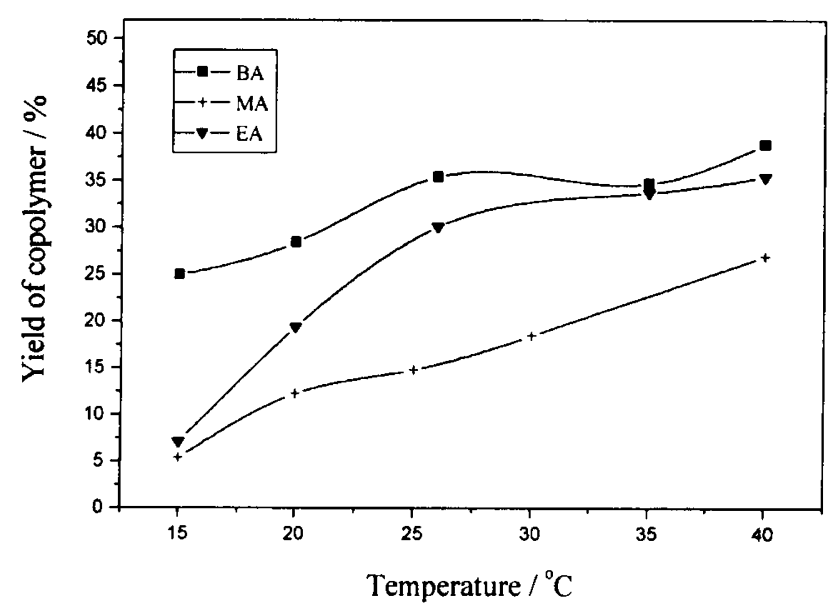

Figure 6. Effects of bulk temperature on yield of copolymer (sonic intensity : $600 \mathrm{w}$; conc. of SDS : $2.5 \%$; conc. of comonomer : $10 \%$; irradiation time : $1 \mathrm{~h} ; V_{\mathrm{A}} / V_{\mathrm{St}}=1$ ).

the SDS oil / water system, $-\mathrm{SO}_{4} \mathrm{Na}$, which extrudes into water phase from the phase interface, is electrolyzed into $\left[-\mathrm{SO}_{4}{ }^{-}\right]$and $\left[\mathrm{Na}^{+}\right]$. The phase interface is surrounded by a layer of $\left[-\mathrm{SO}_{4}{ }^{-}\right]$,which forms a doubleelectron layer with closely orientated arranging $\left[\mathrm{Na}^{+}\right]$. This special structure accelerates the electrophilic free radicals to disperse into phase interface and react with the monomer existing in phase interface to copolymerize. Therefore, the yield of copolymer increases with amount of SDS. Cation surfactant acts in the same way. Acrylate monomer is split into free radicals $\left(\mathrm{CH}_{3} \cdot{ }^{\cdot} \cdot \mathrm{CH}_{2} \mathrm{R}\right.$, and $\mathrm{RO} \cdot$ ). The nucleophilic $\mathrm{CH}_{3} \cdot$, and $\cdot \mathrm{CH}_{2} \mathrm{R}$ are more active than the electrophilic free radical $(\mathrm{RO} \cdot)$ generated in the system and more easily to combine with each other than to disperse out of the cavitation bubble. This results in lower number of free radicals able to disperse out of the cavitation bubbles. Therefore, cation surfactant has less contribution to the copolymerization than anion surfactant. Non-ion surfactant without doubleelectron layer has no effect on the dispersion of free radicals.

Figure 8 shows that as the concentration of comonomer increases the yield of copolymer decreases. This 


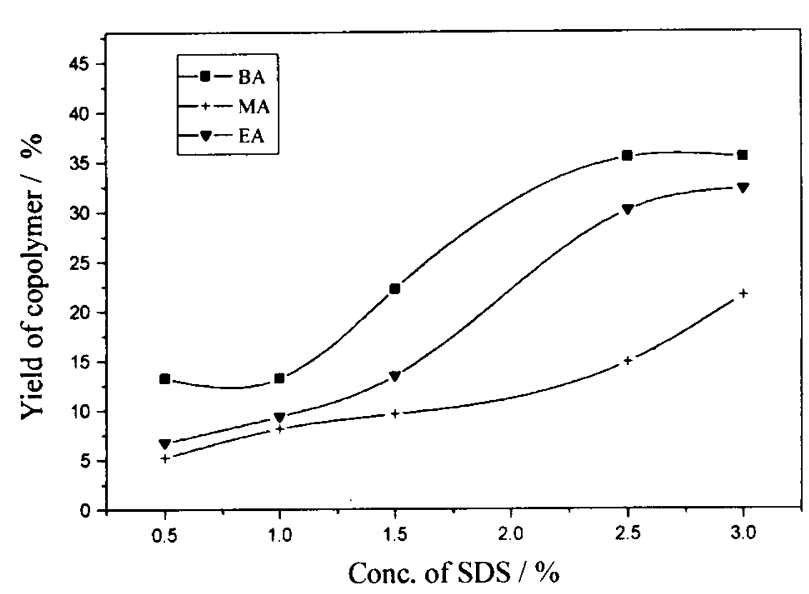

Figure 7. Effects of amount of surfactant on yield of copolymer (sonic intensity : $600 \mathrm{w}$; conc. of comonomer : $10 \%$; irradiation time : $1 \mathrm{~h} ; 25^{\circ} \mathrm{C} ; V_{\mathrm{A}} / V_{\mathrm{St}}=1$ ).

Table II. Effects of surfactant on yield of PS-co-PBA

\begin{tabular}{|c|c|c|c|}
\hline \multirow{2}{*}{ Reaction condition } & \multicolumn{3}{|c|}{ Surfactant } \\
\hline & SDS & CTAB & OP- 10 \\
\hline $25^{\circ} \mathrm{C}, 60 \mathrm{~min}$ & $35.4 \%$ & $18.0 \%$ & 0 \\
\hline $35^{\circ} \mathrm{C}, 60 \mathrm{~min}$ & $34.7 \%$ & $25.2 \%$ & 0 \\
\hline $40^{\circ} \mathrm{C}, 60 \mathrm{~min}$ & $38.8 \%$ & $27.8 \%$ & - \\
\hline $25^{\circ} \mathrm{C}, 45 \mathrm{~min}$ & $20.8 \%$ & $10.8 \%$ & - \\
\hline $25^{\circ} \mathrm{C}, 90 \mathrm{~min}$ & $31.2 \%$ & $15.3 \%$ & 0 \\
\hline
\end{tabular}

Polymerization condition : sonic intensity : $600 \mathrm{w}$; conc. of comonomer : $10 \%$; conc. of SDS : $2.5 \% ; V_{\mathrm{BA}} / V_{\mathrm{St}}=1$.

may result from (1). high monomer concentration lowers relative volume of water phase, leading to decrease of number of micelles able to disperse out of cavitation bubbles (2). with increase of viscosity of the system, the high concentration of comonomer results in fast rise of viscosity, which weakens the effect of cavitation, and lowers the dispersing rate of free radicals.

The ratio of comonomer also influences the yield of copolymer considerably. As Figure 9 shows, increasing the amount of acrylate monomer is helpful to improve the yield of copolymer. When $V_{\mathrm{A}} / V_{\mathrm{St}}$ is $1-1.7$, the yield reaches maximum. During this period, the number of electrophilic free radical generated by cavitation increases, which facilitates the oil/water emulsion copolymerization leading to the rise of yield. That $\mathrm{CH}_{2}$ CHCOOCH${ }_{2} \mathrm{R}$ is more active than $\left\langle-\mathrm{CH}-\mathrm{CH}_{2}\right.$ also contributes to the rise of yield. But when more acrylate monomer is added into the system, the yield drops. This is dependent on the reactivities of monomers. Because acrylate monomer is far less active than St, further increase in their amount influences the equilibrium of the reaction and then lower the yield of copolymerization. Therefore, an optimum ratio of comonomer exists in the system.

\section{CONCLUSIONS}

Copolymers (PS-co-PMA, PS-co-PEA, and PS-co-PBA) are prepared by the sonication of monomers. (St and

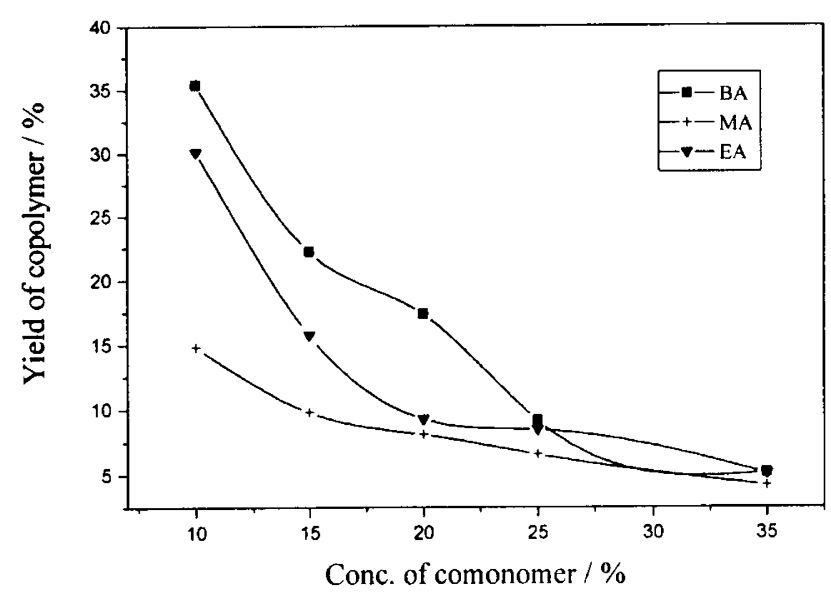

Figure 8. Effects of conc. of comonomer on yield of copolymer (sonic intensity : $600 \mathrm{w}$; conc. of SDS : $2.5 \%$ ' irradiation time : $1 \mathrm{~h}$; $\left.25^{\circ} \mathrm{C} ; V_{\mathrm{A}} / V_{\mathrm{St}}=1\right)$.

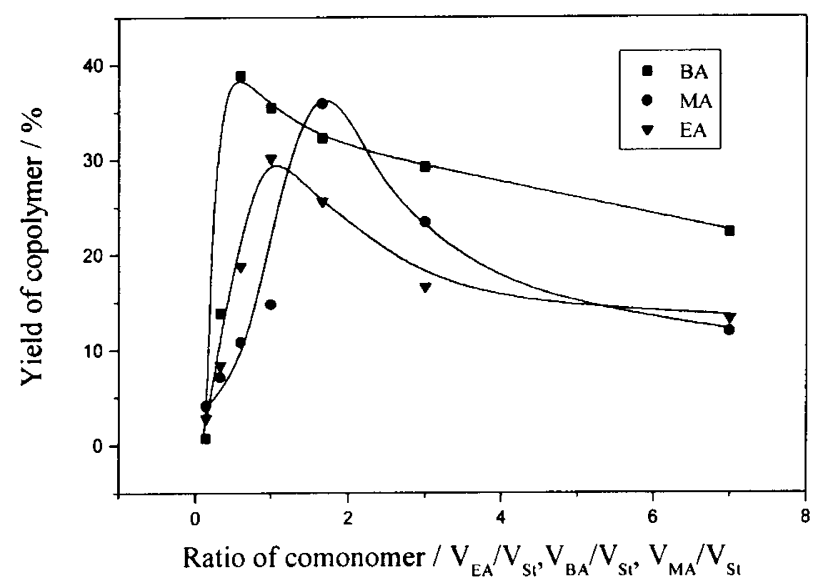

Figure 9. Effects of comonomer ratio on yield of copolymer (sonic intensity : $600 \mathrm{w}$; conc. of comonomer : $10 \%$; conc. of SDS : $2.5 \%$, irradiation time : $1 \mathrm{~h} ; 25^{\circ} \mathrm{C}$ ).

acrylate monomers). The yield of copolymer increased with the rise of ultrasound intensity, reaction time, bulk temperature and the amount of ion surfactant, but decreases with the increase of concentration of comonomer. An optimum ratio of comonomer $\left(V_{\mathrm{A}} / V_{\mathrm{St}}=1-1.7\right)$ exists in the system. The type of surfactant has significant effect on the yield of copolymer. Anion surfactant has more contribution to copolymerization than the cation surfactant. In non-ion surfactant system, no copolymer is obtained.

\section{REFERENCES}

1. D. Peter, J. Mater. Chem ., 6, 1605 (1996).

2. P. Kruus and T. J. Patraboy, J. Phys. Chem., 89, 3379 (1985).

3. G. J. Price, M. R. Daw, N. J. Newcombe, and P. F. Smith, Br. Polym. J., 23, 63 (1990).

4. G. J. Price, D. J. Norris, and P. J. West, Macromolecules, 25, 6447 (1992).

5. R. Feng and H. Li, "Sonochemistry and Its Application", Anhui Press of Science \& Technology, China, 1992, pp 74-88. 